\title{
Fuzzy Detour Convexity and Fuzzy Detour Covering in Fuzzy Graphs
}

\author{
R. Rajeshkumar and A.M. Anto ${ }^{b}$ \\ Research Scholar, Malankara Catholic College, Affiliated to Manonmaniam \\ Sundaranar University, Tamil Nadu, India \\ ${ }^{\mathbf{b}}$ Faculty of Mathematics, Malankara Catholic College, Tamil Nadu, India
}

Article History: Received: 11 January 2021; Accepted: 27 February 2021; Published online: 5 April 2021

\begin{abstract}
A path P connecting a pair of vertices in a connected fuzzy graph is called a fuzzy detour, if its $\mu$ - length is maximum among all the feasible paths between them. In this paper we establish the notion of fuzzy detour convex sets, fuzzy detour covering, fuzzy detour basis, fuzzy detour number, fuzzy detour blocks and investigate some of their properties. It has been proved that, for a complete fuzzy graph G, the set of any pair of vertices in $\mathrm{G}$ is a fuzzy detour covering. A necessary and sufficient condition for a complete fuzzy graph to become a fuzzy detour block is also established. It has been proved that for a fuzzy tree there exists a nested chain of sets, where each set is a fuzzy detour convex. Application of fuzzy detour covering and fuzzy detour basis is also presented.
\end{abstract}

Keywords: fuzzy detour, detour convex, detour covering, detour basis, detour number, fuzzy detour blocks.

\section{Introduction}

It was Lotfi A. Zadeh in 1965 [12] presented the notion of fuzzy sets, which deals with vagueness and lack of exactness in set theory. Later, Prof. Azriel Rosenfeld instilled fuzzy set theory into fuzzy graph theory in 1975 [11]. Infusing real world experience into fuzzy graph will make this area more rejuvenating. Rosenfeld's papers encouraged researchers to think differently and gave them freedom to explore. In fuzzy graphs, he developed fuzzy relations on fuzzy sets and verified graph theoretic concepts. Several other pioneering works in this field can be found in [6] [7] [8].

The notion of detour distance in graphs was presented by Gary Chartrand, Gamy L. Johns and Songlin Tian in [10]. It was Rosenfeld who presented a metric called the $\mu$-distance [11] in fuzzy graphs, later, which was effectively used by various authors in their studies. Dr.A.Nagoorgani and J. Umamaheswari presented the notion of fuzzy detour $\mu$-distance and some of its properties in [1]. Linda and Sunitha extensively studied the notions of $\mu$ - convex sets and its related properties in [3]. This paper serves three purposes. The first one is to establish the notion of fuzzy detour convex set, detour covering, detour basis, detour number and illustrate them with suitable examples. Second purpose is to present the concept of fuzzy detour blocks and some of their characteristics. Finally, to present an application of fuzzy detour covering and fuzzy detour basis.

\section{Preliminaries}

In this paper we use definitions of fuzzy graph [5], fuzzy subgraph [5] and fuzzy complete graph [4]. A fuzzy graph $G:(V, \sigma, \mu)$ is a non-empty set $V$ along with a pair of functions $\sigma: V \rightarrow[0,1]$ and $\mu: E \rightarrow[0,1]$ such that $\forall u, v \in V, \mu(u, v) \leq \sigma(u) \wedge \sigma(v)$. Where the elements in $V$ are the vertices and the elements in $E$ are edges of the fuzzy graph. Let $G:(V, \sigma, \mu)$ is a fuzzy graph, then $H:(V, \tau, \vartheta)$ is called a partial fuzzy subgraph of $G$ if $\tau \subseteq \sigma$ and $\vartheta \subseteq \mu$. Similarly, a fuzzy subgraph $H:(P, \tau, v)$ of $G$ is induced by $P$ if $P \subseteq V, \tau(u)=\sigma(u) \forall u \in P$ and $\vartheta(u, v)=\mu(u, v) \forall u, v \in P$. The support [5] of $\sigma$ is described as, Supp $(\sigma)=\{u \in V: \sigma(u)>0\}$ and is denoted by $\sigma^{*}$.We list some of the definitions and propositions that we use later in our discussion from [5][1][2]

Definition 2.1 In a fuzzy graph $G:(V, \sigma, \mu)$, a path $P$ is a chain of distinct vertices $v_{0}, v_{1} \ldots \ldots v_{n}$ such that $\left(v_{i-1}, v_{i}\right)>0,1 \leq i \leq n$, where $n$ denotes the length of the path.

Definition 2.2 Every path in fuzzy graph $G:(V, \sigma, \mu)$ have a strength and is defined to be $\bigwedge_{i=1}^{n} \mu\left(v_{i-1}, v_{i}\right)$.

Definition 2.3 The maximum of the strength of all paths between a pair of vertices $v_{i}$ and $v_{j}$ is called the strength of connectedness between them and is represented as $\mu^{\infty}\left(v_{i-1}, v_{i}\right)$.

Definition 2.4 A fuzzy graph $G$ : $(V, \sigma, \mu)$ is defined to be a complete fuzzy graph, if $\mu(u, v)=\sigma(u) \wedge \sigma(v)$ $\forall u, v \in \sigma^{*}$.

Definition 2.5 A connected fuzzy graph $G:(V, \sigma, \mu)$ is called a fuzzy tree if it has a fuzzy spanning subgraph $F:(V, \sigma, \tau)$, which is a tree where for all $u v$ not in $F, \mu(u, v)<\tau^{\infty}(u, v)$.

Definition 2.6 Suppose that $G:(V, \sigma, \mu)$ be a connected fuzzy graph. The fuzzy detour $\mu-\operatorname{distance} \Delta(u, v)$ between pair of vertices $u$ and $v$ is defined by the maximum $\mu$ - length of any $u-v$ path, where the $\mu$ - length of a path $P: u=v_{0}, v_{1} \ldots \ldots v_{n}=v$ is 


$$
l(P)=\sum_{i=1}^{n} \frac{1}{\mu\left(v_{i-1}, v_{i}\right)}
$$

A $u-v$ path is called a $u-v$ fuzzy detour if its length is $\Delta(u, v)$.

Proposition 2.7 For the fuzzy detour $\mu$ - distance $\Delta$ on a connected fuzzy graph $G:(V, \sigma, \mu)$, then $(V(G), \Delta)$ is a metric space.

Proposition 2.8 Every fuzzy detour in a complete fuzzy graph is a Hamiltonian path.

Proposition 2.9 A complete fuzzy graph $G$ with $n$ vertices has at least $\frac{n(n-1)}{2}$ distinct fuzzy detour paths.

\section{Fuzzy Detour Convexity}

In fuzzy graph the concept of geodesic convexity and its related properties depending on $\mu$ - distance are introduced in [3]. In this section we establish the notion of fuzzy detour convexity based on fuzzy detour $\mu-$ distance [1] and present the notion of fuzzy detour covering, detour basis, detour number and some of their properties.

Definition 3.1 Let $v_{i}$ and $v_{j}$ be any two vertices in a connected fuzzy graph $G:(V, \sigma, \mu)$, the fuzzy detour closed set is defined to be the set that contains all the vertices in all $v_{i}-v_{j}$ fuzzy detour together with $v_{i}$ and $v_{j}$ and is denoted by $I_{D}\left[v_{i}, v_{j}\right]$

Definition 3.2 In a connected fuzzy graph $G:(V, \sigma, \mu)$, the fuzzy detour closure of $S \subseteq \sigma^{*}$ is defined by the union of all detour closed sets over each pairs of vertices $v_{i}, v_{j} \in S$ and denoted as $I_{D}[S]$.

Definition 3.3 A subset $S$ of $\sigma^{*}$ in a connected fuzzy graph $G:(V, \sigma, \mu)$ is said to be a fuzzy detour convex if its detour closure equals $S$, i.e. $I_{D}[S]=S$.

Example 3.4 For a fuzzy graph $G:(V, \sigma, \mu)$ in Figure 3.1, $\sigma^{*}=\left\{v_{1}, v_{2}, v_{3}, v_{4}\right\}$ and $\mu\left(v_{1}, v_{2}\right)=0.5, \mu\left(v_{2}, v_{3}\right)=$ $0.6, \mu\left(v_{3}, v_{4}\right)=0.5, \mu\left(v_{1}, v_{4}\right)=0.4, \mu\left(v_{1}, v_{3}\right)=0.1$.

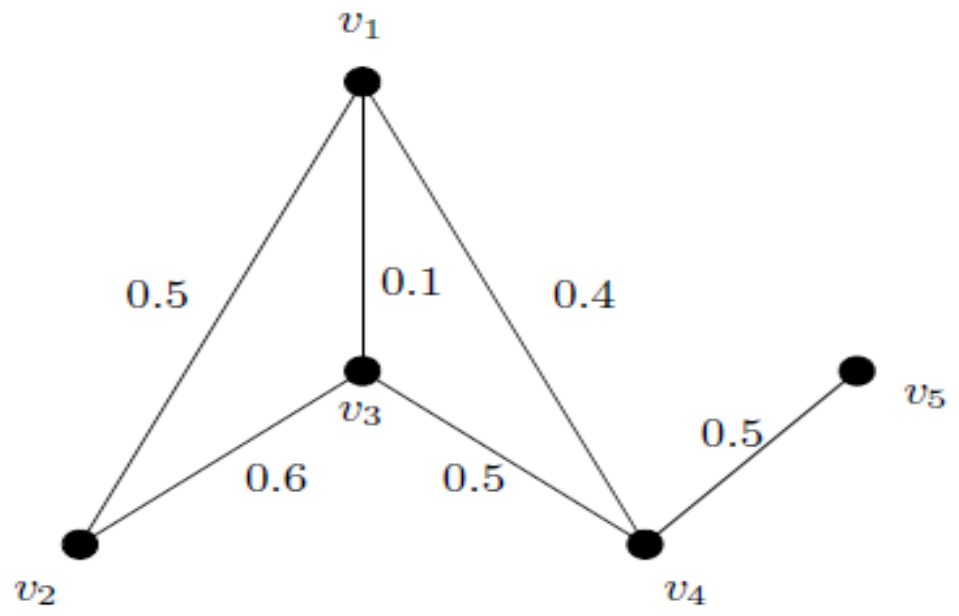

Figure 3.1

Here the path $v_{1}-v_{3}-v_{2}$ is a $v_{1}-v_{2}$ fuzzy detour. The edge $v_{1} v_{3}$ is a $v_{1}-v_{3}$ fuzzy detour. Thus $I_{D}\left[v_{1}, v_{2}\right]$ $=\left\{v_{1}, v_{3}, v_{2}\right\}, I_{D}\left[v_{1}, v_{3}\right]=\left\{v_{1}, v_{3}\right\}, I_{D}\left[v_{1}, v_{4}\right]=\left\{v_{1}, v_{3}, v_{4}\right\}$. If $S_{1}=\left\{v_{1}, v_{2}\right\}$, then $I_{D}\left[S_{1}\right]=\left\{v_{1}, v_{2}, v_{3}\right\} \neq S_{1}$, therefore the set $S_{1}$ is not fuzzy detour convex. But if $S_{2}=\left\{v_{1}, v_{3}\right\}$ then $I_{D}\left[S_{2}\right]=S_{2}$, the set $S_{2}$ is fuzzy detour convex.

Proposition 3.5 The null set $\emptyset$, every singleton sets in $\sigma^{*}$ and the whole vertex set $\sigma^{*}$ for a connected fuzzy graph $G:(V, \sigma, \mu)$ are fuzzy detour convex.

Note 1: If the order of a fuzzy detour convex set $S$ varies over the range $2 \leq O(S)<O\left(\sigma^{*}\right)$, then such set is said to be a nontrivial fuzzy detour convex set.

Theorem 3.6 Suppose $G$ : $(V, \sigma, \mu)$ be a connected fuzzy graph, then the intersection of any two fuzzy detour convex sets is also fuzzy detour convex.

Proof: Assume that $G:(V, \sigma, \mu)$ to be a connected fuzzy graph. Consider any two fuzzy detour convex sets of $\sigma^{*}$, let it be $U$ and $W$. We show that $U \cap W$ is fuzzy detour convex. Let $v_{i}$ and $v_{j}$ be any two vertices in $U \cap W$. Therefore both $v_{i}$ and $v_{j}$ are in $U$ and $W$. Also since $U$ and $W$ are fuzzy detour convex, we have all vertices in all $v_{i}-v_{j}$ fuzzy detours are both in $U$ and $W$. Therefore we have all vertices in all $v_{i}-v_{j}$ fuzzy detours are in $U \cap$ $W$. Hence we are done.

The following example will illustrate the above theorem.

Example 3.7 For the fuzzy graph given in Figure 3.2 


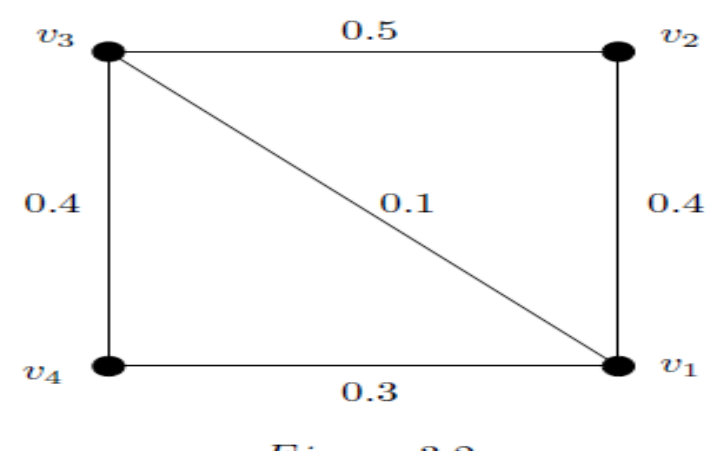

Figure 3.2

We have path $v_{1}-v_{3}-v_{2}$, the edge $v_{1} v_{3}$ and path $v_{2}-v_{1}-v_{3}$ are $v_{1}-v_{2}$ fuzzy detour, $v_{1}-v_{3}$ fuzzy detour and $v_{2}-v_{3}$ fuzzy detour respectively. So $I_{D}\left[v_{1}, v_{2}\right]=\left\{v_{1}, v_{3}, v_{2}\right\}, I_{D}\left[v_{1}, v_{3}\right]=\left\{v_{1}, v_{3}\right\}, I_{D}\left[v_{2}, v_{3}\right]=\left\{v_{2}\right.$, $\left.v_{1}, v_{3}\right\}$. If $S_{1}=\left\{v_{1}, v_{2}, v_{3}\right\}$ then $I_{D}\left[S_{1}\right]=\left\{v_{1}, v_{2}, v_{3}\right\}=S_{1}$. Thus we have $S_{1}$ is a fuzzy detour convex set and if $S_{2}=\left\{v_{1}, v_{3}\right\}$, then $I_{D}\left[S_{2}\right]=S_{2}$, thus $S_{2}$ is also a fuzzy detour convex set. Now $S_{1} \cap S_{2}=\left\{v_{1}, v_{3}\right\}$, which is fuzzy detour convex.

Theorem 3.8 Suppose $G:(V, \sigma, \mu)$ be a fuzzy tree and let $G^{\prime}:\left(V, \sigma^{*}, \mu^{*}\right)$ is a tree. Then we can find a nested chain of sets $\sigma^{*}=V_{n} \supset V_{n-1} \supset V_{n-2} \supset \ldots \ldots . . \supset V_{1}$, where $V_{i}$ is fuzzy detour convex for each $i$.

Proof: Suppose $G:(V, \sigma, \mu)$ be a fuzzy tree and graph $G^{\prime}:\left(V, \sigma^{*}, \mu^{*}\right)$ is a tree with $n$ vertices. Since $G^{\prime}$ is a tree, assures the existence of only one path from one vertex to another vertex. Hence there exist only one fuzzy detour path between every pair of vertices, thus the union of all vertices in all detour path is equal to $\sigma^{*}=V_{n}$, which is fuzzy detour convex and order of $V_{n}$ equals $n$. Let $G_{1}$ be a subgraph of $G$ induced by set $V_{n-1}$, obtained by deletion of a pendant vertex from $V_{n}$. Clearly the induced graph $G_{1}$ is a tree. By similar arguments mentioned above, $V_{n-1}$ is a fuzzy detour convex and order of $V_{n-1}$ equals $n-1$. Proceeding like this till we get a singleton set. Thus we obtained the nested chain of set $\sigma^{*}=V_{n} \supset V_{n-1} \supset V_{n-2} \supset \ldots \ldots \ldots \supset V_{1}$.

Definition 3.9 Suppose a fuzzy graph $G$ : $(V, \sigma, \mu)$ is connected. If $S \subseteq \sigma^{*}$, then $S$ is called a fuzzy detour covering of $G$ if $I_{D}[S]=\sigma^{*}$, (or $I_{D}[S]=V(G)$ )

Definition 3.10 Any fuzzy detour covering of a connected fuzzy graph $G:(V, \sigma, \mu)$ that contains minimum number of vertices is called a minimal fuzzy detour covering of $G$.

Definition 3.11 A fuzzy detour basis for a connected fuzzy graph $G:(V, \sigma, \mu)$ is the minimal fuzzy detour covering that covers $G$.

Definition 3.12 An order of a fuzzy detour basis is the number of vertices in it. This number is so called fuzzy detour number, denoted by $\mu_{n}(G)$.

Example 3.13 For the fuzzy graph $G$ given in Figure 3.3

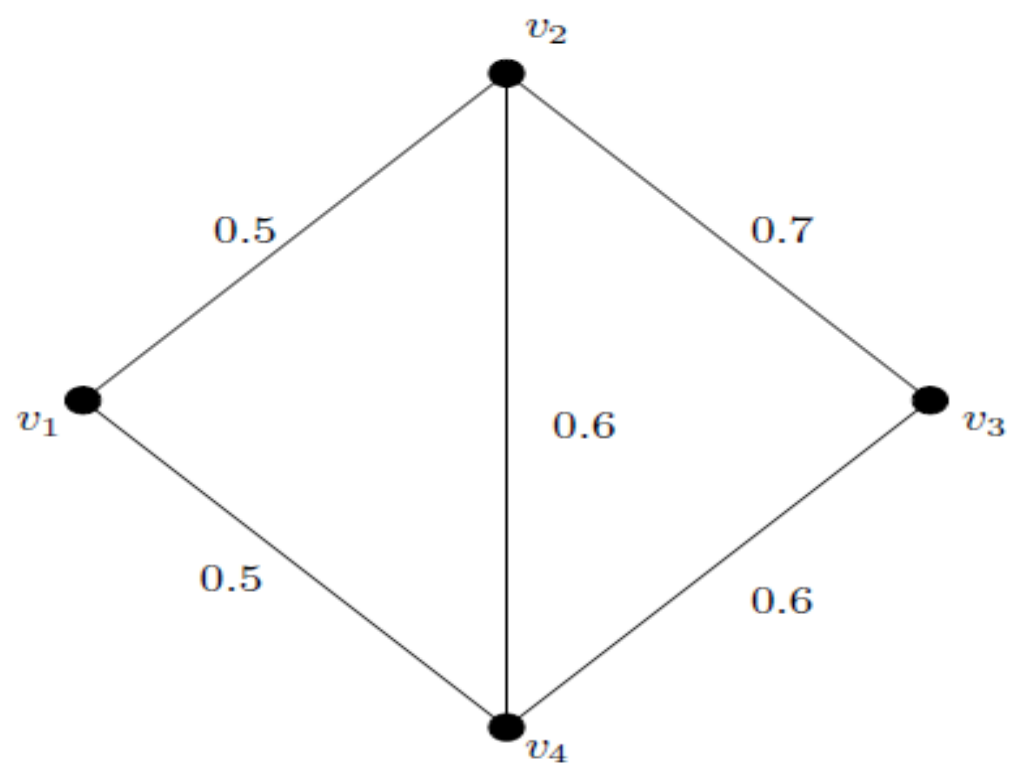

Figure 3.3 
Let $S_{1}=\left\{v_{1}, v_{2}\right\}$, since $v_{1}-v_{4}-v_{3}-v_{2}$ is a $v_{1}-v_{2}$ fuzzy detour path, thus we have $I_{D}\left[S_{1}\right]=\left\{v_{1}, v_{2}, v_{3}, v_{4}\right\}$ $=\sigma^{*}$. Hence $\left\{v_{1}, v_{2}\right\}$ is a fuzzy detour covering of $G$. Similarly $S_{2}=\left\{v_{1}, v_{2}, v_{3}\right\}$ is also a fuzzy detour covering, since we have $I_{D}\left[S_{2}\right]=\left\{v_{1}, v_{2}, v_{3}, v_{4}\right\}=\sigma^{*}$.

Example 3.14 Consider the complete fuzzy graphs $G_{1}$ and $G_{2}$ given in Figure 3.4

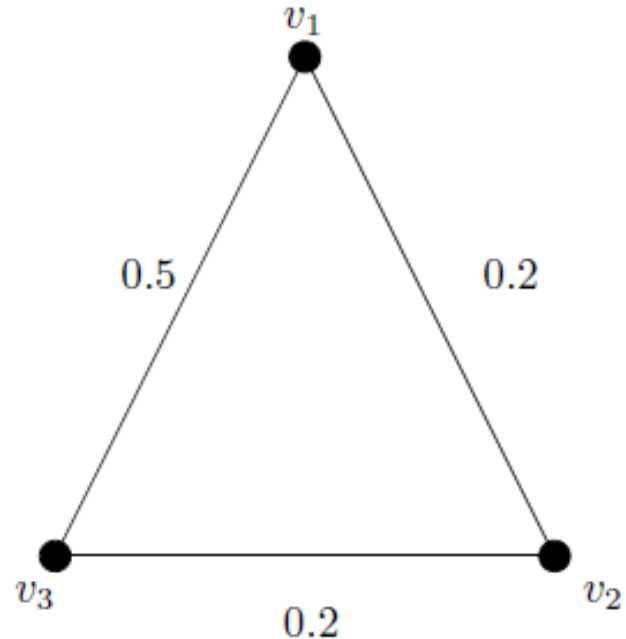

$G_{1}$

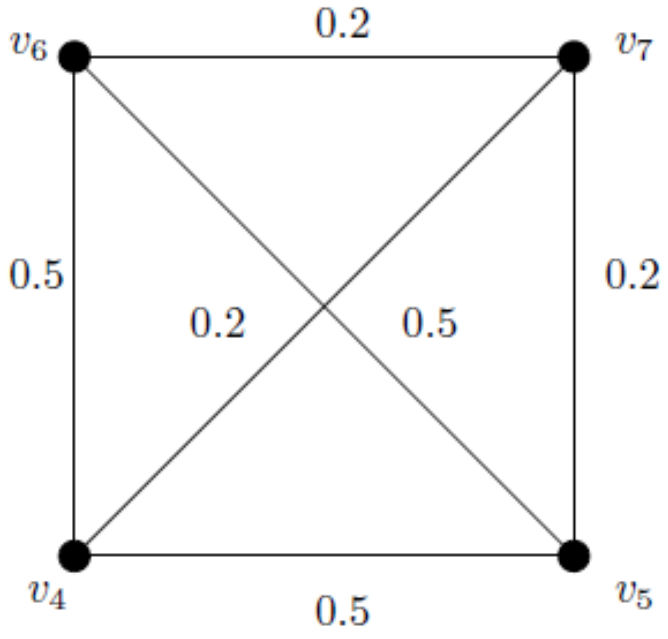

$G_{2}$

\section{Figure 3.4}

In $G_{1}$ we have path $v_{1}-v_{3}-v_{2}$ is a $v_{1}-v_{2}$ fuzzy detour and $\Delta\left(v_{1}, v_{2}\right)=7$

path $v_{1}-v_{2}-v_{3}$ is a $v_{1}-v_{3}$ fuzzy detour and $\Delta\left(v_{1}, v_{3}\right)=10$,

path $v_{2}-v_{1}-v_{3}$ is a $v_{2}-v_{3}$ fuzzy detour and $\Delta\left(v_{2}, v_{3}\right)=7$,

Clearly $\left\{v_{1}, v_{2}\right\},\left\{v_{1}, v_{3}\right\}$ and $\left\{v_{2}, v_{3}\right\}$ are fuzzy detour coverings of $G_{1}$. Also they are the minimal covering that covers $G_{1}$. Hence they are fuzzy detour basis for $G_{1}$.

Similarly in $G_{2}$, path $v_{4}-v_{6}-v_{7}-v_{5}$ is a $v_{4}-v_{5}$ fuzzy detour and $\Delta\left(v_{4}, v_{5}\right)=12$

path $v_{4}-v_{5}-v_{7}-v_{6}$ is a $v_{4}-v_{6}$ fuzzy detour and $\Delta\left(v_{4}, v_{6}\right)=12$

path $v_{4}-v_{5}-v_{6}-v_{7}$ is a $v_{4}-v_{7}$ fuzzy detour and $\Delta\left(v_{4}, v_{7}\right)=9$

path $v_{5}-v_{4}-v_{7}-v_{6}$ is a $v_{5}-v_{6}$ fuzzy detour and $\Delta\left(v_{5}, v_{6}\right)=12$

path $v_{5}-v_{4}-v_{6}-v_{7}$ is a $v_{5}-v_{7}$ fuzzy detour and $\Delta\left(v_{5}, v_{7}\right)=9$

path $v_{6}-v_{4}-v_{5}-v_{7}$ is a $v_{6}-v_{7}$ fuzzy detour and $\Delta\left(v_{6}, v_{7}\right)=9$

Here also $\left\{v_{4}, v_{5}\right\},\left\{v_{4}, v_{6}\right\},\left\{v_{4}, v_{7}\right\},\left\{v_{5}, v_{6}\right\},\left\{v_{5}, v_{7}\right\}$ and $\left\{v_{6}, v_{7}\right\}$ are fuzzy detour covering and detour basis of $G_{2}$.

From the above example 3.14 we have the following observation, that for a complete fuzzy graph $G:(V, \sigma, \mu)$, the set $\left\{v_{i}, v_{j}\right\} \forall v_{i}, v_{j} \in \sigma^{*}$ is a fuzzy detour covering of $G$. This property is generalized from the next theorem.

Theorem 3.15 For a complete fuzzy graph $G:(V, \sigma, \mu)$, the set $\left\{v_{i}, v_{j}\right\}$ is a fuzzy detour covering for any pair of vertices $v_{i}, v_{j} \in \sigma^{*}$.

Proof: Let $G:(V, \sigma, \mu)$ be a complete fuzzy graph. Let $v_{i}, v_{j}$ be any pair of vertices in $\sigma^{*}$. We show that $\left\{v_{i}, v_{j}\right\}$ is a fuzzy detour covering. Since $G$ is complete, by Proposition 2.8 , every fuzzy detour path of a complete fuzzy graph is Hamiltonian [2]. This means, every fuzzy detour traverses through every vertex of $G$ exactly once. Therefore, for any pair of vertices $v_{i}$ and $v_{j}$, we have $I_{D}\left[\left\{v_{i}, v_{j}\right\}\right]=\sigma^{*}$. This proves that $\left\{v_{i}, v_{j}\right\}$ is fuzzy detour covering for any pair of vertices $v_{i}, v_{j} \in \sigma^{*}$.

Corollary 3.16 For a complete fuzzy graph $G:(V, \sigma, \mu),\left\{v_{i}, v_{j}\right\}$ is a fuzzy detour basis of $G$ for any pair of vertices $v_{i}, v_{j} \in \sigma^{*}$.

Proof: Let $G:(V, \sigma, \mu)$ be a complete fuzzy graph. We must show that $\left\{v_{i}, v_{j}\right\}$ is a basis of $G$ for any pair of vertices $v_{i}, v_{j} \in \sigma^{*}$. For that, it is enough to prove that $\left\{v_{i}, v_{j}\right\}$ is a minimal fuzzy detour covering of $G$. By Theorem $3.15\left\{v_{i}, v_{j}\right\}$ is a fuzzy detour covering for any pair of vertices $v_{i}, v_{j} \in \sigma^{*}$. If $\left\{v_{i}, v_{j}\right\}$ is minimal, then we are done. So we assume that there exist a fuzzy detour covering of $G$, say $S$, which is minimal than $\left\{v_{i}, v_{j}\right\}$ 
for any pair of $v_{i}, v_{j} \in \sigma^{*}$. Then $S$ should be a singleton set. Thus $I_{D}[S] \neq \sigma^{*}$, which is a contradiction. Therefore $\left\{v_{i}, v_{j}\right\}$ is a minimal fuzzy detour covering of $G$ for any pair of vertices $v_{i}, v_{j} \in \sigma^{*}$. Thus a basis of $G$.

The next corollary is an instant consequence of above corollary 3.16

Corollary 3.17 Fuzzy detour number, $\mu_{n}(G)$ for a complete fuzzy graph $G:(V, \sigma, \mu)$ of $n$ vertices equals 2 .

\section{Applications}

An application which is closely related is in the field of power supply across wide area. Demand of power can categorise over a particular time period, for example daily, seasonal, occasionally and so on. If we consider a particular city, the energy demand may be different in different areas depending on consumption. Demands may fluctuate on daily, monthly or yearly basis. Usually we prefer to distribute electric energy depending on the demand across different parts effectively and without huge loss of power.

A power station requires an additional electric power supply to meet the demand without failure for a certain time period. But station authorities doesn't have enough time to contact all other power stations. But at the same time authorities were forced for a rapid action in collaboration with all the neighbouring power stations for an additional power supply. Here we can solve this problem by using the concept of fuzzy detour covering. For that we consider a connected fuzzy graph. In particular consider all power stations within a city. Representing the power stations by vertices and transmission lines between them by edges. Every edge there is associated with a membership value (say, resistance level). The reciprocal of membership value represents the flow of current. If an edge have minimum membership value, then the flow of current through it will become high. Power stations may or may not be connected directly all together. More precisely if one power station does not connected the other, then there is no edge correspondence between them.

Consider Figure 4.1 Let $v_{1}, v_{2}, v_{3}, v_{4}, v_{5}$ and $v_{6}$ represents each power stations and $e_{1}, e_{2}, e_{3}, e_{4}, e_{5}, e_{6}, e_{7}, e_{8}$ and $e_{9}$ represents transmission lines between power stations in a particular city.

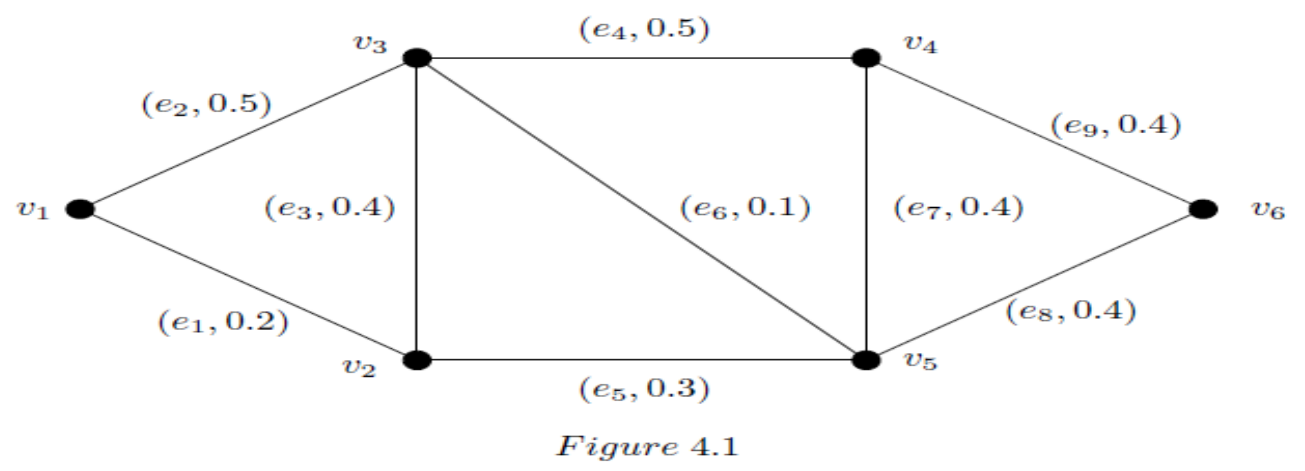

Let us assume that suddenly the power station $v_{6}$ lacking enough power to meet the demand, so it requires emergency power supply from its neighbouring power stations. Now we consider all detour paths between $v_{6}$ and other power stations.

We have path

$P_{1}: v_{6}-v_{4}-v_{5}-v_{3}-v_{2}-v_{1}$ is a fuzzy $v_{6}-v_{1}$ detour path and $\Delta\left(v_{6}, v_{1}\right)=20.83$

$P_{2}: v_{6}-v_{4}-v_{5}-v_{3}-v_{1}-v_{2}$ is a fuzzy $v_{6}-v_{2}$ detour path and $\Delta\left(v_{6}, v_{2}\right)=22$

$P_{3}: v_{6}-v_{4}-v_{5}-v_{2}-v_{1}-v_{3}$ is a fuzzy $v_{6}-v_{3}$ detour path and $\Delta\left(v_{6}, v_{3}\right)=15.3$

$P_{4}: v_{6}-v_{5}-v_{2}-v_{1}-v_{3}-v_{4}$ is a fuzzy $v_{6}-v_{4}$ detour path and $\Delta\left(v_{6}, v_{4}\right)=14.8$

$P_{5}: v_{6}-v_{2}-v_{1}-v_{3}-v_{4}-v_{5}$ is a fuzzy $v_{6}-v_{5}$ detour path and $\Delta\left(v_{6}, v_{5}\right)=14.8$

Here $\left\{v_{6}, v_{1}\right\},\left\{v_{6}, v_{2}\right\},\left\{v_{6}, v_{3}\right\},\left\{v_{6}, v_{4}\right\}$ and $\left\{v_{6}, v_{5}\right\}$ are fuzzy detour covering also they are basis for above fuzzy graph. In fuzzy detour path $P_{2}$ has maximum flow rate, $P_{4}$ and $P_{5}$ has minimum flow rate as compared to other detour paths. So from above details we can conclude that power station $v_{6}$ only to contact any one of other power station for additional power supply. So that power station those who receive message from $v_{6}$ will charge the appropriate detour transmission line depending on demand and pass message to subsequent power stations in this line. In particular, power station $v_{6}$ wants huge load to meet demand, then will opt path $P_{2}$. So power station $v_{6}$ will send request to $v_{2}$ for assistance. Thus $\left\{v_{2}, v_{6}\right\}$ covers all power stations and ensure the required power supply for $v_{6}$. This process can be extended to a wide network. Furthermore, consider every detour distance between all pair of vertices within a particular network, from that find out all detour basis. This detour basis will play an important role in connecting and sharing facilities from one network to another network.

A complete fuzzy graph, fuzzy detour covering and fuzzy detour basis in association with applications of blockchain [13] based solution can considered to be appropriate for reducing intermediaries. Blockchains can broadly be defined as a new form of network infrastructure in the field of information technology that create 'trust' in networks by introducing distributed verifiability, higher level auditability, and general agreements. 
Block chain act like a shared database and thus ensure trust across peer to peer network and also that no individual body can claim the ownership of blockchain network. Moreover no single individual can modify data stored on it or take decision solitarily without the permission from its peers. Here we can apply the application of fuzzy detour covering in blockchain. This may be explored in upcoming work.

\section{Conclusion}

In this paper, we presented the notions of fuzzy detour convex set, detour covering, detour basis and detour number for a fuzzy graph and illustrated them with suitable examples. It has been proved that the intersection of two fuzzy detour convex sets is also a fuzzy detour convex and set of each pair of vertices in a complete fuzzy graph is found to be a detour covering. This paper identifies that the fuzzy detour number for a complete fuzzy graph equals two and for a fuzzy tree there exists a nested chain of sets, where each set is a fuzzy detour convex. Finally, we presented an application of fuzzy detour covering and fuzzy detour basis in electric power transmission.

\section{References}

1. Nagoor Gani,A., and Umamaheswari, J., "Fuzzy Detour $\mu$-Distance in Fuzzy Graphs", Proceeding of the International Conference on Mathematical Methods and Computations, Allied Publications, 2009, pp 184187.

2. Nagoor Gani,A., and Umamaheswari, J., "Fuzzy Detour As A Hamiltonian Path In Fuzzy Graphs "International Journal of Math. Sci. \& Engg. Appls. (IJMSEA) Vol. 5 No. II (March, 2011), pp. 161-166

3. J.P. Linda and M.S. Sunitha, $\mu$ - Geodetic Iteration Number and $\mu$ - Geodetic Number of a Fuzzy Graph, Gen. Math. Notes, Vol. 18, No. 1, ICSRS Publication, 2013, pp.1-12

4. Bhutani, K. R., On automorphisms of fuzzy graphs, Pattern Recognition Letters, 9 (1989) 159-162.

5. Sunil Mathew, John N. Mordeson, Davender S. Malik, "Fuzzy Graph Theory", Springer International Publishing (2018)

6. John N. Mordeson, Sunil Mathew, Advanced Topics in Fuzzy Graph Theory, Springer International Publishing (2019)

7. J.N. Mordeson and P.S. Nair, Fuzzy Graphs and Fuzzy Hypergraphs, Physica - Verlag, (2000).

8. J.N. Mordeson and Y.Y. Yao, Fuzzy cycles and fuzzy trees, The Journal of Fuzzy Mathematics, 10(1) (2002), 189-202.

9. Sameeha Rehmani and M.S. Sunitha, Perfect geodesic fuzzy graphs, International Journal of Pure and

10. Applied Mathematics Volume 120 No. 6 2018, 6243-6251

11. Gary Chartrand, Gamy L. Johns, Songlin Tian, Detour Distance in Graphs, Annals of Discrete Mathem-atics, Elsevier Science Publishers, 55, 127-136 (1993).

12. Rosenfeld, Fuzzy graphs, In: Fuzzy Sets and their Application to Cognitive and Decision Processes, Academic Press, (1975).

13. Zadeh.L.A., Fuzzy sets, Information and Control, 8(1965), 338-353.

14. Michael Crosby, Nachiappan, Pradan Pattanayak, Sanjeev Verma, Vignesh Kalyanaraman BlockChain Technology: Beyond Bitcoin, Applied Innovation Review, Issue No. 2, June 2016 